\title{
Correlation Between Orthodontic Forces and Root Resorption - a Systematic Review of the Literature
}

\author{
Alexandru Vlasa, Lia Yero Eremie, Luminița Lazăr, Anamaria Bud, Mariana Păcurar, Eugen Bud, \\ Carmen Biriș
}

University of Medicine and Pharmacy, Tîrgu Mureș, Romania

\section{CORRESPONDENCE}

\section{Lia Yero Eremie}

Str. Gheorghe Marinescu nr. 38

540139 Tîrgu Mureș, Romania

Tel +40 265215551

E-mail: liayero@yahoo.ro

\section{ARTICLE HISTORY}

Received: 17 June, 2016

Accepted: 22 August, 2016
Alexandru Vlasa • Str. Gheorghe Marinescu nr. 38, 540139 Tîrgu Mureș, Romania. Tel +40 26521555 Luminița Lazăr • Str. Gheorghe Marinescu nr. 38, 540139 Tîrgu Mureș, Romania. Tel +40 265215551 Anamaria Bud • Str. Gheorghe Marinescu nr. 38 540139 Tîrgu Mureș, Romania. Tel +40 265215551 Mariana Păcurar • Str. Gheorghe Marinescu nr. 38 540139 Tîrgu Mureș, Romania. Tel +40 26521555 Eugen Bud • Str. Gheorghe Marinescu nr. 38, 540139 Tîrgu Mureș, Romania. Tel +40 265215551

Carmen Biris - Str. Gheorghe Marinescu nr. 38, 540139 Tîrgu Mureș, Romania. Tel +40 265215551

\begin{abstract}
Orthodontically induced external apical root resorption (OIEARR) is a major concern regarding periodontal status after nonsurgical orthodontic treatment. The aim of this study was to assess this sequel by a systematic review of published data. For assessment, we performed an electronic search of one database for comprehensive data, using keywords in different combinations: "root resorption", "periodontics" and "nonsurgical orthodontic treatment". We supplemented the results searching by hand in published journals and we cross-referenced with the accessed articles. Patients included in the results presented a good general health status, with no previous history of OIEARR and no other associated pathologies. Finally, twenty-three studies were selected and included in this review. A high prevalence (69-98\%) and moderate severity of OIEARR ( $<5 \mathrm{~mm}$ and $<1 / 3$ from original root length) were reported. No difference in root resorption was found regarding the sex of the patients. A moderate positive correlation between treatment duration and root resorption was found. Also, a mild correlation regarding antero-posterior apical displacement and root resorption was found.
\end{abstract}

Keywords: root resorption, orthodontics, periodontal disease

\section{INTRODUCTION}

Orthodontic treatment ensures proper alignment of the teeth and improves the occlusal and jaw relationship. Like any other treatment modalities, orthodontic treatment, in addition to its benefits, has also associated risks and complications. ${ }^{1}$ The only risk factors that have been supported by previous evidence will be reviewed in this article.

Root resorption is defined as the destruction of the cementum or dentin by cementoclastic or osteoclastic activity; it may result in the shortening or blunting of the root. ${ }^{2}$ It is an inflammatory process resulting in an ischemic necrosis in the periodontal ligament when the orthodontic force is applied. ${ }^{1}$ Root resorption occurs when the pressure on the cementum exceeds its reparative capacity 
and dentin is exposed, allowing the multinucleated odontoclasts to degrade the root substance. ${ }^{3}$

The etiology of root resorption still remains unclear and is complex, including genetic predisposition and environmental factors. ${ }^{4}$ Orthodontically induced external apical root resorption (OIEARR) is a sterile inflammatory process that is extremely complex. ${ }^{2}$ The loss of apical root structure is not predictable; when it progresses reaching the dentine, it is considered irreversible. Severe root resorption after orthodontic treatment compromises the outcome of successful orthodontic treatment. ${ }^{1}$

This review aims to highlight the main coordinates of risk issues of root resorption in orthodontics.

\section{MATERIALS AND METHODS}

All the protocols regarding reports for systematic reviews of health sciences studies were respected in accordance with the PRISMA statement.

\section{Data sources}

Comprehensive searches up to March 31, 2016 were used on the PubMed electronic bibliographic database (1972 to March 2016, week 5). The keywords used for this literature search were "root resorption", "periodontics" and "orthodontics". Only human studies were eligible, the initial search retrieved 1024 articles, and from the retrieved articles, manual searches were subsequently performed. No restrictions were applied regarding publication year or journal category.

\section{Study design}

The articles that were selected encompass the following inclusion-exclusion criteria:

Population - individuals with large over-jets and crowding were included, with no restrictions regarding gender. Patients included in the results presented a good general health status, with no previous history of OIEARR and no other associated pathologies.

Treatment - patients undergoing orthodontic treatment by removable or fixed appliances.

Outcome - root resorption assessed by the root lengths of maxillary teeth using OPT x-ray, before and after treatment.

In the first part of the review process, adequate abstracts were selected and after this process only twenty-three articles met the inclusion criteria.

\section{RESULTS}

The search yielded twenty-three potential studies for inclusion from the electronic database. Full texts of these journal articles were retrieved for examination.

\section{Sex and age}

Most of the studies included both male and female patients; however, there is no evidence suggesting any differences in root resorption between genders. ${ }^{6}$ Four studies examined young teenagers with a mean age of 13 years. ${ }^{6-9}$

\section{Treatment duration}

Many studies confirmed a higher risk and severity of apical root resorption in patients with an increased duration of orthodontic treatment. ${ }^{9-16}$ However, other authors found no statistical significant association between root resorption and treatment duration..$^{17,18}$

\section{Appliance type}

Fixed appliances have been shown to cause more root resorption than removable appliances, which can be explained by the increased range of tooth movement afforded by fixed appliances. ${ }^{19}$ The risk of root resorption associated with different bracket designs has yielded inconclusive results. ${ }^{20,21}$ It is generally agreed that the use of a rapid maxillary expander is associated with increased levels of root resorption. ${ }^{22-25}$

\section{Treatment mechanics}

L. Linge and B.O. Linge suggested that the use of intermaxillary elastics increased the amount of root resorption, but Sameshima and Sinclair did not find any correlation. ${ }^{19,26}$

\section{Force magnitude}

More recent studies have confirmed that the higher forces increase the amount of external root resorption, thus confirming the previous studies. ${ }^{27,28}$ Reitan, on the other hand, found that external root resorption was poorly correlated with the force magnitude. ${ }^{29}$

\section{Force duration}

Debate exists as to whether more root resorption is associated with continuous or intermittent forces. Many believe that discontinuous forces produce less root resorption be- 
cause the pause in tooth movement allows the resorbed cementum to heal. ${ }^{30-35}$ Acar et al. examined 22 human teeth. The patients were exposed to a continuous tipping force of $100 \mathrm{~g}$ on one side, and on the other side an intermittent force was applied through elastics for 12 hours per day over a period of 9 weeks. Their results showed that the intermittent forces resulted in less root resorption. The accuracy of these results is questionable because the intermittent forces were subject to patient compliance. ${ }^{34}$

Weiland studied 84 premolars, which had been moved buccally with an orthodontic appliance. ${ }^{35}$ On one side of the mouth, force on the premolar was applied with a stainless steel wire (0.016 inch), while force on the contralateral premolar was applied with a superelastic wire (0.016 inch). Their results support the findings of Acar et al. that continuous forces cause more resorption. ${ }^{36}$ They showed that the teeth activated with the super elastic wire had moved significantly more, but had $140 \%$ more resorption than the teeth with stainless steel wire. Contrary to these reports, Owman-Moll et al. found no difference in the amount or severity of root resorption between forces applied continuously or intermittently after the application of a buccally directed force of $50 \mathrm{~g}$ to human premolars. ${ }^{36}$

\section{Direction of tooth movement}

Intrusion has been consistently implicated as the most likely type of tooth movement to cause root resorption. ${ }^{16,29}$ Displacement of the root apex horizontally or torquing has been proven beyond doubt to produce root resorption. ${ }^{26,37}$ Reitan and Thilander et al. suggested that the stress distribution associated with tipping movements is more likely to cause root resorption than the stress distribution associated with bodily movement. 25,30

\section{Amount of tooth movement}

Sameshima and Sinclair found that severe root resorption occurred in their samples when the root apex was displaced lingually, with a mean difference of $1 \mathrm{~mm}$ more than the control group. ${ }^{26}$ They concluded that root resorption is directly related to the distance moved by the tooth roots. Maxillary incisors tend to be moved more than other teeth in orthodontic treatment, and therefore this is a possible explanation for why maxillar incisors have a higher risk of root resorption.

\section{Extraction}

Sameshima and Sinclair examined the relationship of the extraction pattern in detail as a factor affecting the resorp- tion process. ${ }^{26}$ They observed a statistically significant difference in the resorption process when extraction and non-extraction groups were compared; among the extraction groups, the extraction of all first premolars showed the greatest resorption potential. Other studies that examined this factor did not find it to be significant. ${ }^{38,39}$

\section{DISCUSSIONS}

Orthodontic treatment is usually contraindicated in patients with active periodontal disease or poor periodontal health, as the chance of further periodontal deterioration is high in such cases. The current literature evidence available for orthodontically induced external root resorption is conflicting and inconclusive.

\section{CONFLICT OF INTERESTS}

The authors declare that there is no conflict of interests regarding the publication of this paper.

\section{REFERENCES}

1. Alfuriji S, Alhazmi N, Alhamlan N, et al The Effect of Orthodontic Therapy on Periodontal Health. International Journal of Dentistry. 2014;2014:6. Article ID 585048

2. Scheibel PC, Ramos AL, Iwaki LCV, Micheletti KR. Analysis of correlation between initial alveolar bone density and apical root resorption after 12 months of orthodontic treatment without extraction. Dental Press Journal of Orthodontics. 2014;19(5):97-102.

3. Jung $\mathrm{Y}-\mathrm{H}$, Cho B-H. External root resorption after orthodontic treatment: a study of contributing factors. Imaging Science in Dentistry. 2011;41(1):17-21.

4. Al-Qawasmi RA1, Hartsfield JK Jr, Everett ET. Genetic predisposition to external apical root resorption. Am J Orthod Dentofacial Orthop. 2003;123(3):242-252

5. Tieu LD, Saltaji H, Normando D, Flores-Mir C. Radiologically determined orthodontically induced external apical root resorption in incisors after non-surgical orthodontic treatment of class II division 1 malocclusion: a systematic review. Prog Orthod. 2014;15:48.

6. DeShields RW. A study of root resorption in treated Class II. Division malocclusions. Angle Orthod. 1969;39(4):231-245.

7. Hollender L, Rönnerman A, Thilander B. Root resorption, marginal bone support and clinical crown length in orthodontically treated patients. Eur J Orthod. 1980;2(4):197-205.

8. Reukers EA, Sanderink GC, Kuijpers-Jagtman AM, van't Hof MA Radiographic evaluation of apical root resorption with 2 different types of edgewise appliances. Results of a randomized clinical trial. J Orofac Orthop. 1998;59(2):100-109.

9. Taner T, Ciger S, Sencift Y. Evaluation of apical root resorption following extraction therapy in subjects with Class I and Class II malocclusions. Eur J Orthod. 1999;21(5):491-496.

10. Martins DR, Tibola D, Janson G. Effects of intrusion combined with anterior retraction on apical root resorption. Eur J Orthod. 2012;34(2):170-175.

11. Sunku R, Roopesh R, Kancherla P, Perumalla KK, Yudhistar PV, Reddy VS Quantitative digital subtraction radiography in the assessment of external apical root resorption induced by orthodontic therapy: a retrospective study. J Contemp Dent Pract. 2011;12(6):422-428.

12. Kinzinger GS, Fritz UB, Sander FG, Diedrich PR. Efficiency of a pendulum appliance for molar distalization related to second and third molar eruption stage. Am J Orthod Dentofacial Orthop. 2004;125:8-23.

13. Wahab MA, Dasor R, Senafi M, et al. Crevicular alkaline phosphatase activity and rate of tooth movement of female orthodontic subjects under different continuous force applications. International Journal of Dentistry. 2013;[245818]. 
14. McFadden WM, Engstrom C, Engstrom H, Anholm JM. A study of the relationship between incisor intrusion and root shortening. Am J Orthod Dentofacial Orthop. 1989;96(5):390-396.

15. VonderAhe G. Postretention status of maxillary incisors with root-end resorption. Angle Orthod. 1973;43(3):247-255.

16. Dermaut LR, De Munck A. Apical root resorption of upper incisors caused by intrusive tooth movement: a radiographic study. Am J Orthod Dentofacial Orthop. 1986;90(4):321-326.

17. Campos MJ, Silva KS, Gravina MA, Fraga MR, Vitral RW. Apical root resorption: the dark side of the root. Am J Orthod Dentofacial Orthop. 2013;143(4):492-498.

18. Weltman B, Vig KW, Fields HW, Shanker S, Kaizar EE. Root resorption associated with orthodontic tooth movement: a systematic review. Am J Orthod Dentofacial Orthop. 2010;137(4):462-476.

19. Linge L, Linge $B O$. Patient characteristics and treatment variables associated with apical root resorption during orthodontic treatment. Am J Orthod Dentofacial Orthop. 1991;99(1):35-43.

20. Malmgren O, Goldson L, Hill C, Orwin A, Petrini L, Lundberg M. Roo resorption after orthodontic treatment of traumatized teeth. Am J Orthod. 1982;82(6):487-491.

21. Mavragani M, Vergari A, Selliseth NJ, Bøe OE, Wisth PL. A radiographic comparison of apical root resorption after orthodontic treatment with a standard edgewise and a straight-wire edgewise technique. Eur J Orthod. 2000;22(6):665-674.

22. Barber AF, Sims MR. Rapid maxillary expansion and external roo resorption in man: a scanning electron microscope study. Am J Orthod. 1981;79(6):630-652.

23. Odenrick L, Karlander EL, Pierce A, Kretschmar U. Surface resorption following two forms of rapid maxillary expansion. Eur $J$ Orthod. 1991;13(2):264-270.

24. Vardimon AD, Graber TM, Voss LR, Lenke J. Determinants controlling iatrogenic external root resorption and repair during and after palatal expansion. Angle Orthod. 1991;61(2):113-122.

25. Thilander B, Rygh $P$, Reitan $K$. Tissue reactions in orthodontics. Orthodontics: Current Principles and Techniques. Ed. Graber T. 2005

26. Sameshima GT, Sinclair PM. Predicting and preventing root resorption: Part I. Diagnostic factors. Am J Orthod Dentofacial Orthop. 2001;119(5):505-510.
27. Chan E, Darendeliler MA. Physical properties of root cementum. Part 5. Volumetric analysis of root resorption craters after application of light and heavy orthodontic forces. Am J Orthod Dentofacial Orthop. 2005;127(2):186-195.

28. Harris DA, Jones AS, Darendeliler MA. Darendeliler, Physical properties of root cementum. Part 8. Volumetric analysis of root resorption craters after application of controlled intrusive light and heavy orthodontic forces: a microcomputed tomography scan study. Am J Orthod Dentofacial Orthop. 2006;130(5):639-647.

29. Reitan K. Initial tissue behavior during apical root resorption. Angle Orthod. 1974;44(1):68-82

30. Reitan K. Effects of force magnitude and direction of tooth movement on different alveolar bone types. The Angle Orthodontist. 1965;34(4):244255.

31. Dougherty HL. The effect of mechanical forces upon the mandibular buccal segments during orthodontic treatment. Am J Orthod. 1968;54(2):83-103.

32. Oppenheimer SJ, Snodgrass GJ. Neonatal rickets. Histopathology and quantitative bone changes. Arch Dis Child. 1980;55(12):945-949.

33. Levander $E$, Malmgren $O$, Eliasson S. Evaluation of root resorption in relation to two orthodontic treatment regimes. A clinical experimental study. Eur J Orthod. 1994;16(3):223-228.

34. Acar A, Canyürek U, Kocaaga M, Erverdi N. Continuous vs. discontinuous force application and root resorption. Angle Orthod. 1999;69(2):159-163.

35. Weiland F. Constant versus dissipating forces in orthodontics: the effect on initial tooth movement and root resorption. Eur J Orthod. 2003;25(4):335342

36. Owman-Moll P, Kurol J, Lundgren D. The effects of a four-fold increased orthodontic force magnitude on tooth movement and root resorptions. An intra-individual study in adolescents. Eur J Orthod. 1996;18(3):287-294.

37. Parker RJ, Harris EF. Directions of orthodontic tooth movements associated with external apical root resorption of the maxillary central incisor. Am J Orthod Dentofacial Orthop. 1998;114(6):677-683.

38. Kaley J, Phillips C. Factors related to root resorption in edgewise practice. Angle Orthod. 1991;61(2):125-32.

39. Baumrind S, Korn EL, Boyd RL, Maxwell R. The decision to extract. Part II. Analysis of clinicians' stated reasons for extraction. Am J Orthod Dentofacial Orthop. 1996;109(4):393-402. 